\title{
Robotic Geometric and Volumetric Inspection of High Value and Large Scale Aircraft Wings
}

\author{
Carmelo Mineo, Charles MacLeod, Riliang Su, Dave \\ Lines, Santi Davì, Bruce Cowan and S. Gareth Pierce \\ Dept. of Electronic and Electrical Eng., University of \\ Strathclyde, Glasgow, United Kingdom \\ carmelo.mineo@ strath.ac.uk
}

\author{
Scott Paton, Gavin Munro, Coreen McCubbin and \\ David Watson \\ Spirit AeroSystems (Europe) Limited \\ Prestwick, United Kingdom
}

\author{
William Kerr and Richard Millar \\ Advanced Forming Research Centre, Strathclyde University, Renfrew, United Kingdom.
}

\begin{abstract}
Increased demands in performance and production rates require a radical new approach to the design and manufacturing of aircraft wings. Performance of modern robotic manipulators has enabled research and development of fast automated non-destructive testing (NDT) systems for complex geometries. This paper presents recent outcomes of work aimed at removing the bottleneck due to data acquisition rates, to fully exploit the scanning speed of modern 6-DoF manipulators. The geometric assessment of the parts is carried out with a robotised dynamic laser scanner encoded through an absolute laser tracker. This method allows scanning speeds up to $330 \mathrm{~mm} / \mathrm{s}$ at $1 \mathrm{~mm}$ pitch. State of the art ultrasonic instrumentation has been integrated into a large robot cell to enable fast data acquisition, high scan resolutions and accurate positional encoding. A fibre optic connection between the ultrasonic instrument and the server computer enables data transfer rates up to $1.6 \mathrm{~GB} / \mathrm{s}$. The robotic inspection system presented herein is currently being tested for industrial exploitation. The adopted system integration strategies allow traditional ultrasonic phased array scanning as well as full matrix capture (FMC) and other novel scanning approaches (e.g. multi-Tx phased array). Scan results, relative to a $1.2 \mathrm{~m} \times 3 \mathrm{~m}$ carbon fibre sample, are presented. The system shows a reference scanning rate of $25.3 \mathrm{~m}^{2} /$ hour with an $8 \mathrm{Tx} / 8 \mathrm{Rx}$ PA approach and an ultrasonically reachable scanning rate over $100 \mathrm{~m}^{2} /$ hour with the novel techniques.
\end{abstract}

Keywords-Robotic NDT; Phased array; Laser scanning; CFRP

\section{INTRODUCTION}

The aerospace industry faces, more than other sectors, the need to speed up the inspection of critical components. To cope with future demand projections for modern aircrafts, it is essential to overcome the current NDT bottlenecks. Manual scanning is a slow process for large samples and requires trained technicians. Moreover, it can be challenging to obtain repeatable results in structures where complex inspection setups are required [1]. Developing reliable automated solutions has become an industrial priority to speed up repetitive inspections in the production chain.

Semi-automated inspection systems have been developed to overcome some of the shortcomings of the manual inspection techniques, using both mobile and fixed robotic platforms. For a number of years, the use of linear manipulators and bridge designs has provided the most stable conditions in terms of positioning accuracy [2]. The use of these systems to inspect parts with non-complex shapes (plates, cylinders or cones) is widespread. Typically, they are specific machines which are used to inspect identically shaped and/or sized parts. In the spectrum of robot manipulators, some modern robots have suitable attributes to develop automated NDT systems and cope with the challenging requirements posed by the aerospace industry [3]. They include precise mechanics and the ability to export positional data at frequencies up to $1 \mathrm{kHz}$. Some applications of 6-axis robotic arms in the NDT field have been developed and published during the last few years, resulting in a growing interest in using such automation solutions [3-6].

Despite these previous efforts, there remain challenges to be addressed. Current research is tackling key challenges: the generation of flexible and adaptive robot tool-paths, the development of high speed NDT data collection and the integration of surface metrology measurements. New data visualization and fast data analysis methods are required to truly speed up the overall throughput of the inspection process.

Traditionally, NDE and metrology measurements are undertaken at different stages of a product manufacture cycle using specific dedicated equipment and personnel. However, since both processes involve direct interaction with the component's surface, motivations exist to combine NDE and metrology to potentially reduce overall cycle time. The collaboration between the University of Strathclyde and Spirit Aerosystems is establishing an automated hybrid cell demonstrator, which is capable of both ultrasonic NDT and metrology inspection. This paper focuses on the NDT development carried out within the project. Emphasis is given to the ultrasonic (UT) instrumentation and the way it has been interfaced to the robotics.

This work was funded by Spirit AeroSystems (Prestwick, UK). 


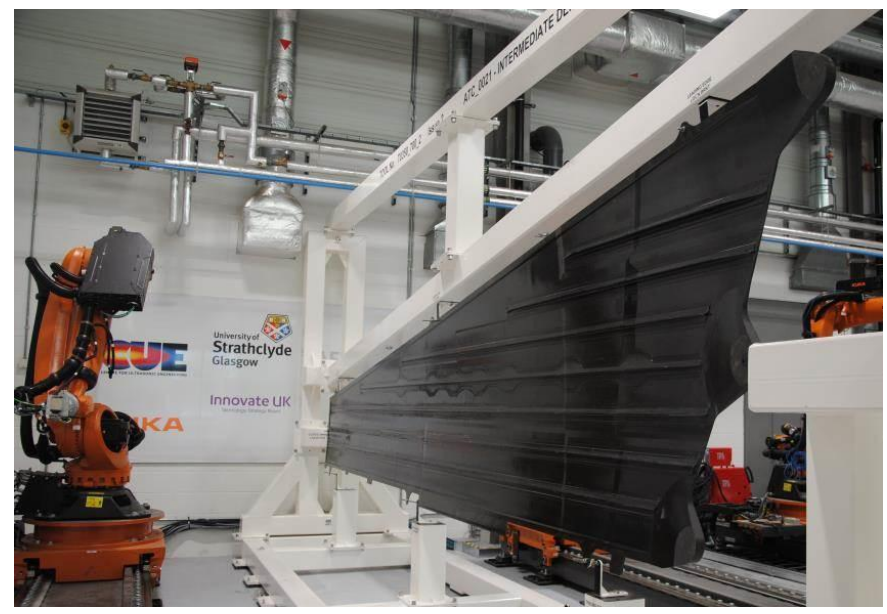

Fig. 1. Multi-robot flexible inspection at the University of Strathclyde

\section{SYSTEM DESCRIPTION}

\section{A. Robotics}

The robotic inspection cell is shown in Fig. 1. It comprises two industrial robots, mounted on linear tracks. A custom tooling frame was purposely designed to support a 7-metre long carbon fibre reinforced plastic (CFRP) aircraft wing sample. The cell occupies an area of $60 \mathrm{~m}^{2}$, surrounded by fixed safety guarding with access gates.

\section{B. Laser scanning}

A dynamic laser scanner is used together with a laser tracker to assess the geometry of the parts. The laser scanning head is capable of scanning the sample at rates of up to $330 \mathrm{~mm} / \mathrm{s}$ (with $1 \mathrm{~mm}$ pitch). The combination of laser scanning head and laser tracker has given promising results on a range of surfaces, from matte black to highly reflective areas.

\section{Ultrasonic transducer}

Traditional methods to perform UT scans of components require the use of a single-element probe or a multiplexed group of probes. Ultrasonic single-element (SE) and phased array (PA) testing have been integrated into several robotic inspection system prototypes over recent years [6-8]. Since the ultrasonic probes require coupling with the surface of the part under inspection, these prototypes have used purposely developed water jet nozzles that embed the transducer and provide a water column for the transmission of the ultrasonic beam.

This work enables robotic ultrasound inspection by means of an ultrasonic array probe housed within a rubber coupled wheel sensor. This approach was originally developed for faster manual inspection techniques of large components. It removes the need for a free water column. Such rubber coupled wheel sensor embeds a 64-element, $0.8 \mathrm{~mm}$ pitch transducer. The wheel probe comes with an ergonomically designed handle to allow manual inspection. In our integration, the wheel probe was removed from the handle and mounted onto Robot \#1, through a purposely designed support (see

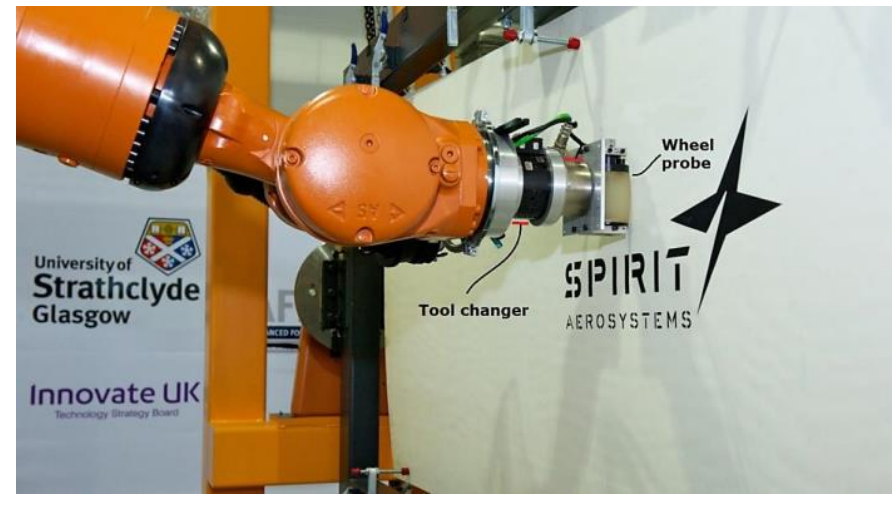

Fig. 2. Rubber coupled wheel sensor mounted onto the robot through a support.

\section{Data acquisition instrument}

The typical portable ultrasonic instruments are designed for delivery of manual scanning and their limited memory is not suitable to collect large high-resolution C-scan images, which is essential for the automated inspection of large aerospace components. Moreover, the data acquisition rate of such devices is a bottleneck that would limit the scanning speed to values well below the maximum speed of industrial 6-DoF manipulators. Additionally, portable instruments have limited features for data encoding and only 2D representations of the results are typically available.

A customized instrument was designed and developed. This is herein referred as FIToolbox instrument. The instrument enables high data rates by exploiting the flexibility of Field Programmable Gate Arrays (FPGAs) in a standard PC platform [9]. The FPGAs can be reconfigured under software control to provide a flexible platform, handling applications from array imaging to multi-channel automated inspection systems. The FIToolbox system uses off-the-shelf modules, here configured as 64 channels of high-speed analogue acquisition with high performance FPGAs for real-time, deterministic processing. It is a 64Tx/64Rx system that interfaces to the ultrasonic array probe via multi-channel pulser-receivers with custom FPGA code. The instrument enables high performance multi-channel ultrasound acquisition and processing, while being flexible enough to address a wide range of research applications. FIToolbox is modular and the independent nature of the transmitter and receiver modules means that both pulse-echo and throughtransmission modes are supported.

The data transfer rate of high performance phased array receivers is often limited by the connection between the device and the controlling computer. This is not the case for the FIToolbox, where a PCI Express bus provides the highest bandwidth of all PC I/O buses. A high speed data transfer cable option allows data streaming at up to $1.6 \mathrm{~GB} / \mathrm{s}$ into the PC. The instrument was mounted the carriage of Robot \#1 within a protective splash proof cover that provides Ingress Protection (IP) rate 53.

\section{E. Integration}

In order to enable the high level of automation required by the current industrial demands, fundamental software has been developed to establish robust communications between the robot controllers, the metrology equipment, the NDT instrumentation 
and the server computer. $\mathrm{C \#}$ and $\mathrm{C}++$ programming language were chosen to develop crucial software components. These languages allow a low level control of the communication strategies and are suitable to develop data acquisition algorithms that run in a reliable manner. The crucial modules were compiled in Dynamic Link Libraries (DLL). The robotic DLL, integrated in the data acquisition FIToolbox platform, enables the use of the robot positional feedback to encode the ultrasound data.

\section{OPTIONS FOR HIGH SPEED IMAGING}

The FIToolbox data collection software platform contains several modules. The robotic control software was integrated with the FIToolbox's streaming module. This module allows for Full Matrix Capture (FMC), where the matrix of transmitting and receiving elements has to be fully populated and only one transmitter is fired at a time. FMC is the most demanding method, for the required transfer speed.

With pulse repetition frequency (PRF) of $10 \mathrm{kHz}$ and a 64-el. probe, the theoretical rate of frames per second (FPS) is $156 \mathrm{~Hz}$ $(10 \mathrm{kHz} / 64)$. The desired resolution in the scanning direction $(r)$ limits the scan speed (s). For a resolution of $1 \mathrm{~mm}$ and at a resulting data transfer rate of $1.55 \mathrm{~GB} / \mathrm{s}$ (still within the limit of $1.6 \mathrm{~GB} / \mathrm{s}), s=r \cdot F P S=156 \mathrm{~mm} / \mathrm{s}$.

Acquiring the data and beamforming inside the FPGAs does not give significant frame rate increase as it is limited by PRF (fixed by transit time) and number of lines. A 64-el. probe with a transmitting/receiving sub-aperture of 8 elements produces 57 lines/frame, giving $12 \%$ increase in the maximum scanning speed $(64 / 57=1.12)$. Two data acquisition strategies have been identified for enabling high speed ultrasonic imaging, herein referred as paintbrush and multi-Tx beam acquisition and beamforming.

\section{A. Paintbrush}

The method is based on the use of a data capture strategy with transmission aperture set to the full array. This means only 1 pulse per frame is required, resulting in the frame rate being equal to the PRF $(10 \mathrm{kHz})$, with no data rate change as still 64 channels/pulse are collected. The method allows a theoretical scanning speed equal to $s=r \cdot P R F$. Maintaining $r=1 \mathrm{~mm}$, $s=10 \mathrm{~m} / \mathrm{s}$. However, all elements fired at once generate a wide flat wave front and there is no focusing on the beam profile.

\section{B. Multi-Tx beam acquisition and beam-forming}

An 8-element sub-aperture results to be the optimum aperture, since the aperture width matches the probe elevation of $6.4 \mathrm{~mm}$ and gives a square excited area, thus the best beam shape. For the 64-el. array embedded in the rubber housed probe, the basic idea consists in enabling the acquisition of a complete frame to produce 57 beams from just 8 pulses. This can be done through parallel firing of multi-Tx sub-apertures along the array, possibility enabled by the FIToolbox hardware in use. There is no change in the required acquisition data rate, as still 64 channels/pulse would be acquired. This method gives a speedup factor of $57 / 8=7.125$ when compared to the single aperture transmission. The multi-Tx beam acquisition allows exploiting the FPGAs for beam-forming. The 8 pulses per frame at $10 \mathrm{kHz}$ PRF produces a frame acquisition time of $800 \mu$ s. The maximum theoretical robot scanning speed is $1.25 \mathrm{~m} / \mathrm{s}$ and the 8 transmissions are offset by $125 \mu \mathrm{m}$ in the scan direction on the reconstructed C-scan. This should still be acceptable for acoustic alignment of Tx and Rx paths.

\section{RESULTS}

\section{A. Quantitative performance comparison}

A commercial scanner performing SE pulse-echo inspection and a system performing PA pulse-echo inspection, both based on 3-DoF gantry probe manipulation, were tested. The first system was capable of reaching a scanning rate of $3.6 \mathrm{~m}^{2} /$ hour (assuming a $1 \mathrm{~mm}$ step between parallel raster scan passes). The second system benefitted from the wide PA probe active area, showing scanning rate of $8.1 \mathrm{~m}^{2} /$ hour.

Table 1 compares the acquisition rate of the 3-DoF scanners with the scanning rates of the system. The anisotropy of CFRP makes it behave as a collimator and reduces the effect of focusing. This means that the paintbrush results are similar to the multi-Tx results, and faster. This is the reason why the multiTx c-scan is not shown. The initial scan trials were carried out with a simple linear PA sweep of 1-element Tx/Rx across the active axis of the array. The paintbrush FRD approach was also tested.

The system shows a scanning rate of $25.3 \mathrm{~m}^{2} /$ hour with the pulse-echo $8 \mathrm{Tx} / 8 \mathrm{Rx}$ approach and an ultrasonically reachable scanning rate of $117.6 \mathrm{~m}^{2} /$ hour with the pulse-echo paintbrush approach. The first method is 2.3 times faster than the PA 3-DoF scanner and 5.6 times faster than the SE system. The paintbrush method and the multi-Tx approach result respectively 13.5 times and 9.4 times faster than the PA 3-DoF scanner.

TABLE I. SCANNING RATES OF COMMERCIAL 3-DOF SCANNERS AND 6DOF NOVEL ROBOTIC SYSTEM.

\begin{tabular}{|l|c|c|c|c|c|}
\hline & \multicolumn{2}{|c|}{ 3-DoF scanners } & \multicolumn{3}{|c|}{ Robotic NDT system } \\
\hline Type of inspection & SE & PA & PA & PA & PA \\
\hline Probe & & $\begin{array}{c}64 \mathrm{el}- \\
1.0 \mathrm{~mm}\end{array}$ & \multicolumn{3}{|c|}{ 64el - 0.8mm } \\
\hline Data acquisition strategy & & $\begin{array}{c}\text { Beam- } \\
\text { forming }\end{array}$ & $\begin{array}{c}8 \mathrm{Tx} \\
8 \mathrm{Rx}\end{array}$ & Paintbrush & $\begin{array}{c}\text { Multi- } \\
\text { Tx }\end{array}$ \\
\hline Sub-aperture & 1 & 10 & 8 & $57 \mathrm{Tx} / 8 \mathrm{Rx}$ & 8 \\
\hline Scanning res. $(\mathrm{mm})$ & 1 & 1 & 1 & 1 & 1 \\
\hline Theoretical FPS $(\mathrm{Hz})$ & 10000 & 185.18 & 174.72 & 10000 & 1250 \\
\hline FPS (Hz) & 1000 & 44 & 154 & 715 & 500 \\
\hline Max scan speed (mm/s) & 1000 & 44 & 154 & 715 & 500 \\
\hline Scanning step (mm) & 1 & 54.98 & 45.6 & 45.6 & 45.6 \\
\hline Scan rate $\left(\mathrm{m}^{2} / \mathrm{hour}\right)$ & 3.6 & 8.7 & 25.3 & 117.6 & 82.1 \\
\hline
\end{tabular}

\section{B. Multi-Aperture Excitation}

The multi-Tx approach is applied to increase the frame rate, which is to use more apertures in a single pulse. The main concern with multi-aperture excitation is the interference between the neighbouring apertures. In order to reduce the interference level between the apertures, a gap can be inserted between two nearby apertures. The number of pulses per frame for multi-aperture is determined by the number of elements in the aperture $\left(\mathrm{N}_{\text {aper }}\right)$, the total number of elements in the array $\left(\mathrm{N}_{\text {array }}\right)$ and the number of gap elements between apertures $\left(\mathrm{N}_{\text {gap }}\right)$. A multi-aperture experiment was carried out on a $75 \mathrm{~mm}$ steel specimen (Fig. 3) with a $3 \mathrm{~mm}$ side drilled hole (SDH), using 8-element aperture by changing the gap from 0 to 8 
elements. The focus point was set to be fixed at $12.5 \mathrm{~mm}$. The linear phased array, was a $5 \mathrm{MHz}$ centre frequency probe with 32 elements and $0.7 \mathrm{~mm}$ pitch. The array was in direct contact with the steel block. The excitation waveform was $40 \mathrm{~V}$, single cycle, tri-level square wave.

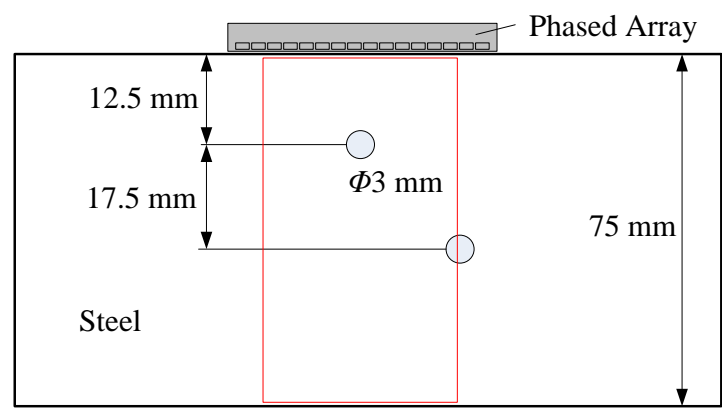

Fig. 3. Schematic diagram of experiment setup on a steel block, the red rectangle is the B-scan image area.

The first B-scan image in Fig. 4 is acquired using linear beamforming and is herein referred as the reference result. The other images are relative to the multi-aperture excitation with gap elements from 0 to 8 . The echo on the right side of the Bscan image is the reflected signal of the neighbouring hole with a different depth. Focusing on the indication of the hole in the middle, the interference of the neighbouring beams is evident when there is no gap between each transmit aperture. As the gap between the apertures increases, the interference level reduces at the expense of the number of pulses.

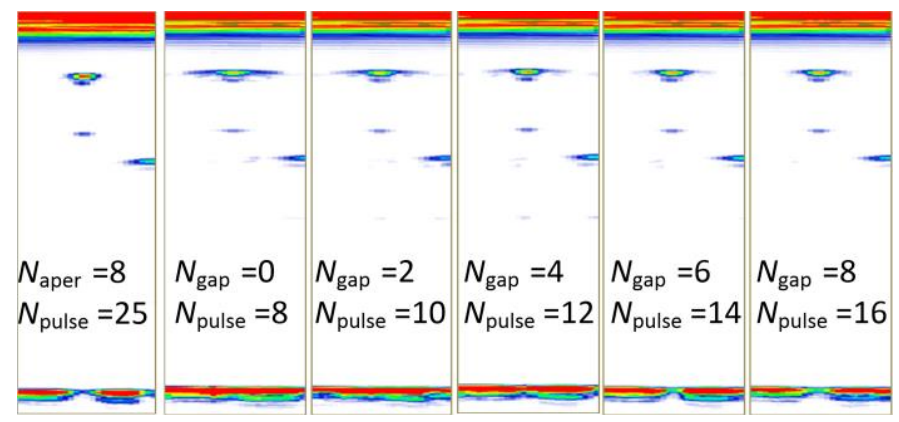

Fig. 4. B-scan images for linear beamforming and multi-aperture excitation with different gaps.

\section{C-scans}

A sub-scale wing cover section, measuring approximately $1.2 \mathrm{~m} \times 3 \mathrm{~m}$ and of thickness varying from 6 to $18 \mathrm{~mm}$, has been scanned to test the system. It was a CFRP part with stringers, cocured to the inner mould line.

A full reference manual scan was acquired through the standard configuration of the rubber housed probe, linked to an OmniScan instrument. To overcome the OmniScan limited operating memory of circa $300 \mathrm{MB}$, the sample surface was partitioned in four regions that were scanned separately. Two calibrated string encoders were used to produce $x-y$ coordinates by triangulation. The partitioning of the sample surface allowed achieving a resolution of $1.6 \mathrm{~mm}$ along the phased array probe axis (using a 2-el. step of the PA sub-apertures) and $2 \mathrm{~mm}$ in the scanning direction. The four sub-scans were downloaded to a computer and merged through the InspectionWare software, developed by UTEX Scientific Ltd. Fig. 5 shows the resulting merged depth map.

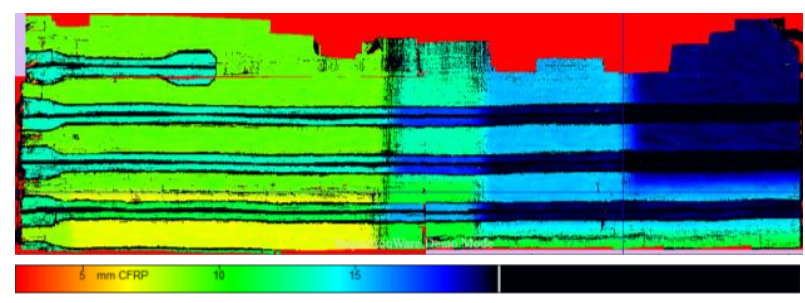

Fig. 5. Merged depth map resulting from manual scan.

The sample was scanned twice through the system, firstly using the $8 \mathrm{Tx} / 8 \mathrm{Rx}$ method and secondly with the paintbrush method (57Tx/8Rx). Higher amount of UT energy penetrates the sample when using the paintbrush approach, since all array elements are triggered at once, producing higher back wall reflections. The robot positional feedback, used to encode the NDT data, produces 3D NDT maps free of misalignments. Fig. 6 shows the top view of the obtained c-scans, both with resolution of $0.8 \mathrm{~mm}$.

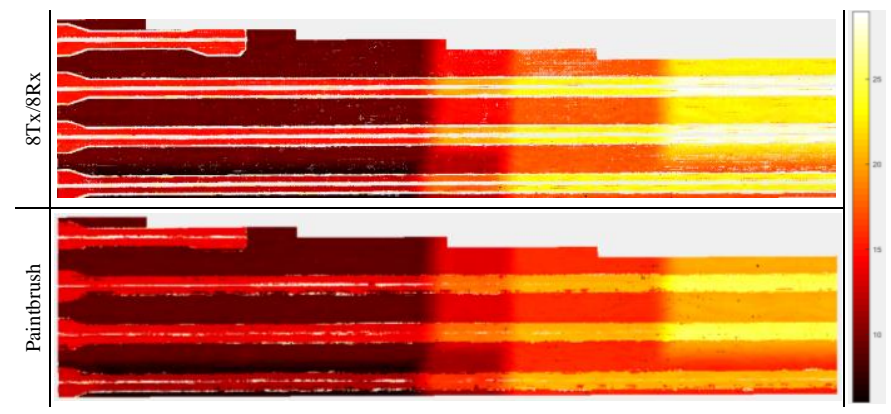

Fig. 6. Depth maps obtained using the robotic system.

\section{CONCLUSIONS}

High acquisition rates are required to fully exploit the speed of modern 6-DoF manipulators. State of the art UT instrumentation was integrated into a large robot cell to enable fast data acquisition, high scan resolutions and accurate positional encoding to obtain 3D scan maps. Two data acquisition strategies were identified for enabling high speed UT imaging and exploiting the $1.6 \mathrm{~GB} / \mathrm{s}$ transfer rate, enabled by fast data transfer connection between the FIToolbox instrument and the server computer. The robotic inspection system prototype shows, to date, a scanning rate of $25.3 \mathrm{~m}^{2} /$ hour with the $8 \mathrm{Tx} / 8 \mathrm{Rx}$ approach and an ultrasonically reachable scan rate over $100 \mathrm{~m}^{2} /$ hour with the paintbrush approach. The robot speed was intentionally limited to $150 \mathrm{~mm} / \mathrm{s}$ for the results described in this paper. Faster robot manipulation requires optimization of the force torque sensor control to cope with the more challenging dynamic constraints given by high speed. The data rate onto disk can become the bottleneck on practical implementations of paintbrush \& Multi-Tx configurations. High-speed drives and data compression techniques are being considered to remove this issue. This optimization work and the software implementation of the multi-Tx beam-forming acquisition method is currently on-going.

Current research is focusing on innovative imaging algorithms to enable the ultrasonic imaging of challenging areas 
(e.g. radii, stringer feet and corners) from the main skin surface. Moreover, software modules for assisted defect recognition and big data analysis is under development.

\section{ACKNOWLEDGMENT}

We acknowledge Dr. R. Freemantle (Wavelength NDT Ltd) for acquiring the manual reference scan.

\section{REFERENCES}

[1] T. Sattar, "Robotic non-destructive testing," Industrial Robot: An International Journal, vol. 37, 2010.

[2] P. Louviot, A. Tachattahte, and D. Garnier, "Robotised UT Transmission NDT of Composite Complex Shaped Parts," presented at the 4th International Symposium on NDT in Aerospace, Berlin (Germany), 2012.

[3] E. Cuevas, M. López, and M. García, "Ultrasonic Techniques and Industrial Robots: Natural Evolution of Inspection Systems," presented at the 4th International Symposium on NDT in Aerospace, Berlin (Germany), 2012.
[4] F. Bentouhami, B. Campagne, E. Cuevas, T. Drake, M. Dubois, T. Fraslin, et al., "LUCIE - A flexible and powerful Laser Ultrasonic system for inspection of large CFRP components.," presented at the 2nd International Symposium on Laser Ultrasonics, Talence (France), 2010.

[5] J. T. Stetson and W. D. Odorico, "Robotic inspection of fiber reinforced aerospace composites using phased array UT," presented at the 40th Annual Review of Progress in Quantitative NDE, Baltimore, Maryland, 2013.

[6] C. Mineo, S. Pierce, B. Wright, I. Cooper, and P. Nicholson, "PAUT inspection of complex-shaped composite materials through six DOFs robotic manipulators," Insight-Non-Destructive Testing and Condition Monitoring, vol. 57, pp. 161-166, 2015.

[7] Robotic Non Destructive Inspection NSpect 210. Available: http://genesissystems.com/products/nspect-systems - Accessed 30/6/2016

[8] Tecnatom, "Tecnatom passes the acceptance tests for two new aeronautical systems," NDT.net e-Journal, vol. 19, 2013.

[9] D. Lines, J. Wharrie, and J. Hottenroth, "Multi-channel ultrasound toolbox: A flexible modular approach for real-time array imaging and automated inspection," in ASNT Conference, Houston, 2010, pp. 15-18. 